\title{
Discrimination of short temporal intervals: A comparison of two models
}

\author{
DAVID J. GETTY \\ Brown University, Providence, Rhode Island 02912
}

\begin{abstract}
Duration-discrimination data from an experiment using empty auditory intervals in a two-alternative forced-choice paradigm are presented. The observed functional relationship between standard deviation of the psychometric density function and stimulus duration is shown to be fit significantly better by a Weber's law model of duration discrimination than by Creelman's counter model. Both models fail to predict the rapid rise in the Weber fraction observed for durations longer than about 2 sec. However, the Weber's law model, based on a generalization of Weber's law, accurately predicts the initial drop in the Weber fraction for very short durations and the observed constancy of the Weber fraction for durations up to 2 sec.
\end{abstract}

Weber's law has endured as a useful empirical description of the relationship between discriminability and stimulus magnitude for a number of intensive sensory dimensions (Holway \& Pratt. 1936; Luce \& Galanter. 1963: Miller, 1947). However, in reviewing a number of studies of duration discrimination, both Allan and Kristofferson (1974) and Woodrow (1951) conclude that Weber's law does not hold for duration. The studies they cite cover a range of durations from $.63 \mathrm{msec}$ to $30 \mathrm{sec}$. While the evidence they present argues strongly that their conclusion is correct for that entire range of durations, the possibility remains that Weber's law, or perhaps a generalized form, holds for some more limited range.

Several recent studies of human duration discrimination (Abel, 1972a, b; Kinchla, 1972) have provided some support for a counter model of human timing first proposed by Creelman (1962). Whereas Creelman tested durations from .25 to $.80 \mathrm{sec}$ and found that the counter model predicted discriminability well over the entire range, Abel (1972a) concluded that the model did reasonably well only for durations less than about .1 sec, failing for longer durations. On the other hand. Kinchla (1972) found support for the counter model over a range of 1 to 8 sec.

In this paper, the counter model and a Weber's law model, based on a generalization of Weber's law, are outlined and several predicted functions derived. The two models are then compared in terms of their ability to account for the data from a duration discrimination experiment also reported here.

This research was supported by Public Health Service Grant MH24143 to the author. I wish to express my gratitude to Marilyn Adams and Seth Roberts for their helptul comments and suggestions, and especially to Russell Church for his critical discussions of this research with me on numerous occasions. I also wish to thank Tom Wing for his help in designing the experiment and his participation as a subject. Requests for reprints should be sent to David J. Getty. Psychology Department. Brown University. Providence, Rhode Island 02912.

\section{Weber's Law Model}

In its strict form. Weber's law asserts that the increment in duration, $\Delta T$, required to produce a fixed but arbitrary level of discriminability is a constant proportion of the standard duration, T. For later convenience, we may choose as our level of discriminability that probability value such that $\Delta \mathrm{T}$ corresponds to the standard deviation of the psychometric density function. The term psychometric density function is defined as that probability function which has the psychometric function as its cumulative distribution function. This measure may be contrasted with the difference limen, which is defined as one-half the interquartile range of the psychometric density function. For a normal distribution, the value of the difference limen is a constant fraction (.68) of the value of the standard deviation. With the standard deviation chosen as our measure of dispersion, Weber's law is given as $\mathrm{SD}(\mathrm{T})$ $=\mathrm{k}_{\mathrm{W}} \mathrm{T}$, where $\mathrm{k}_{\mathrm{w}}(>0)$ is the Weber fraction. Thus, if Weber's law holds, we predict that the variance of the psychometric density function will grow as the square of $T$, a faster than linear growth:

$$
\operatorname{Var}_{W^{\prime}}(T)=\left(k_{W} T\right)^{2} \text {. }
$$

It should be noted that it is the standard deviation of the psychometric density function which is asserted to obey Weber's law, rather than the standard deviation of any hypothetical underlying distribution of internal duration. The reason for not specifying a particular model of the internal representation of duration is that there are several such models which predict that the standard deviation of the psychometric density function obeys Weber's law, at least one of which does not assume that Weber's law applies to the internal distribution. In particular, the Weber"s law prediction can be shown for a model which assumes (a) that the mean internal duration is 
given by the logarithm of external duration and (b) that the standard deviation of the distribution of internal values is a constant. independent of the external duration. On the other hand, Weber's law is also predicted to hold for the psychometric function by a model which assumes (a) that a linear relationship holds between mean internal and external duration, and (b) that Weber's law applies to the standard deviation of the distribution of internal values. Thus, the general Weber's law model assumed here actually describes a class of models defined by the property that the predicted standard deviation of the psychometric density function obeys Weber's law. In addition. some assumption must be made concerning the shape of the psychometric function in order to obtain an estimate of the standard deviation. We will assume that the normal density function provides a good first approximation to the true psychometric density function.

If Weber's law holds, then the ratio $\mathrm{SD}(\mathrm{T}) / \mathrm{T}$, the Weber fraction, should be a constant, independent of $T$. Failure of the Weber fraction to remain constant has been observed both for very short durations and for relatively long durations. Using empty intervals in a two-alternative forced-choice paradigm. Blakely (1933; cited in Woodrow, 1951) obtained a Weber fraction which was a broad U-shaped function of standard duration. The fraction was relatively constant at a value between .08 and .10 for durations between 0.2 and $1.5 \mathrm{sec}$, but rose for both shorter and longer durations. Similar results were obtained by Stott (1933; cited in Woodrow, 1951) for filled durations.

In fact, a number of duration-discrimination studies have also reported data which ind icate a rising Weber fraction for decreasing duration below .2 sec (Abel, 1972a. b; Allan, Kristofferson, \& Wiens, 1971; Creelman, 1962; Small \& Campbell, 1962). Moreover, this same phenomenon has been observed in connection with discrimination along a number of intensive sensory dimensions (Holway \& Pratt, 1936; Miller. 1947). The most common explanation is in terms of a sensory noise component which is independent of the stimulus magnitude and always present. Fechner proposed a generalized Weber's law to account for the initial drop in the Weber fraction, given by the equation $S D=k(T+c)$, where $c$ is the constant noise magnitude.

Fechner's generalization would appear to be difficult to justify on other than empirical grounds. If the effective stimulus is the sum of a sensory magnitude and an independent noise magnitude, then the distribution of their sum is given by the convolution of the distributions of the sensory and noise components. It is the variances of the two components that will be additive rather than the standard deviations, as suggested by Fechner's generalization. This leads to a somewhat different generalization of Weber's law. We may suppose that variability in a discrimination task arises from several sources, some of which are dependent upon the stimulus magnitude and others of which are independent of stimulus magnitude. Assuming the mutual independence of these sources, the total variance will be the sum of the component variances. We may lump all magnitudedependent variance into one component, $V(T)$, and all magnitude-independent variance into a constant residual component, $V_{R}$. Thus, total variance, $\operatorname{Var}(T)$, for a duration $T$ is given by the sum

$$
\operatorname{Var}(T)=V(T)+V_{R}
$$

If $V(T)$ obeys Weber's law (Equation 1), then Equation 2 becomes

$$
\operatorname{Var}_{W}(T)=k_{W}^{2} T^{2}+V_{R}
$$

and

$$
\mathrm{SD}_{\mathrm{W}}(\mathrm{T})=\left(\mathrm{k}_{\mathrm{W}}{ }^{2} \mathrm{~T}^{2}+\mathrm{V}_{\mathrm{R}}\right)^{1 / 2}
$$

This function is significantly nonlinear only for small values of $T$, and has an intercept value of $V_{R}{ }^{1 / 2}$. For large $T$, the residual variance, $V_{R}$ becomes negligible, and the standard deviation approaches a straight line having slope $\mathrm{k}_{\mathrm{W}}$ and zero intercept.

The generalized Weber fraction is then given by

$$
\frac{\operatorname{SD}_{W}(T)}{T}=\left(k_{W}^{2}+\frac{V_{R}}{T^{2}}\right)^{1 / 2}
$$

As $T$ increases from zero, the Weber fraction decreases rapidly, approaching a constant value of $\mathrm{k}_{\mathrm{w}}$.

The generalization of Weber's law and the Weber fraction given in Equations 4 and 5, respectively, will be used in evaluating the Weber's law model.

\section{Counter Model}

The counter model proposed by Creelman (1962) assumes that the internal, or subjective, representation of a duration is the integral of a series of pulses emitted by one or more stochastic sources at a fixed mean rate. These pulses are integrated only during the duration to be timed. Creelman further assumed that the pulse source is a random emitter. As a consequence, the total number of pulses accumulated in an interval, $\mathrm{T}$, is described by the Poisson distribution with mean and variance both equal to $\lambda T$. where $\lambda$ is the mean rate of pulse emission.

In order to apply the basic counting mechanism to two-alternative forced-choice data, Creelman as- 
sumed a decision mechanism based on signal detection theory. For the standard duration $T$ and the comparison duration $T+\Delta T$, the subject is assumed to obtain independent counts, $C_{\mathbf{T}}$ and $C_{T+\Delta T}$, respectively, and to report as longer that interval having the larger count. For $\lambda \mathrm{T}$ large, the Poisson distribution of the count $C_{T}\left(C_{T+\Delta T}\right)$ is closely approximated by a normal distribution with mean and variance equal to $\lambda T[\lambda(T+\Delta T)]$. The distribution of the difference between the counts, $D=$ $\mathrm{C}_{\mathrm{T}+\Delta \mathbf{T}}-\mathrm{C}_{\mathrm{T}}$, will therefore be approximately normal with mean $\lambda \Delta T$ and variance $\lambda(2 T+\Delta T)$. The probability of judging the comparison duration as longer is given by the area under the distribution of $D$ for $D$ greater than some criterion value, which determines the point of subjective equality. For fixed $\mathrm{T}$, as $\Delta \mathrm{T}$ is varied continuously from negative to positive values, a plot of the probability of judging the comparison longer (the psychometric function) will trace out an asymmetric ogive with a steeper slope for negative $\Delta \mathrm{T}$ than for positive $\Delta \mathrm{T}$. The asymmetry is a consequence of the fact that the variance of the comparison count distribution increases with $\Delta \mathrm{T}$. Of primary importance, however, is the observation that for $\Delta T$ equal to 0 the variance of the distribution of count differences is directly proportional to the standard duration $\mathrm{T}$ :

$$
\operatorname{Var}_{C}(T)=k_{C} T \text {, }
$$

where $\mathrm{k}_{\mathrm{C}}(>0)$ is the constant of proportionality for the counter model.

The argument made in the context of the Weber's law model regarding a possible residual variance component can also be made here. Allowing this possibility, the equation for total variance becomes

$$
\operatorname{Var}_{\mathbf{C}}(T)=k_{C} T+V_{R}
$$

Thus, the counter model predicts a linear growth of variance with increasing duration, compared to the nonlinear growth predicted by the Weber's law model.

Finally, the Weber fraction predicted by the counter model may be derived:

$$
\operatorname{SD}_{C}(T)=\left(k_{C} T+V_{R}\right)^{1 / 2}
$$

and

$$
\frac{\mathrm{SD}_{\mathrm{C}}(\mathrm{T})}{\mathrm{T}}=\left(\frac{\mathrm{k}_{\mathrm{C}}}{\mathrm{T}}+\frac{\mathrm{V}_{\mathrm{R}}}{\mathrm{T}^{2}}\right)^{1 / 2}
$$

Thus, whereas the Weber's law model predicts that the Weber fraction will approach $\mathrm{k}_{\mathrm{W}}$ as $\mathrm{T}$ increases, the counter model predicts that the Weber fraction will be continuously decreasing towards an asymptote of zero.
In order to provide data to adequately test these two models. an experiment $w$ as run with a dense set of 15 standard durations in the range from 50 to $3.200 \mathrm{msec}$.

\section{METHOD}

\section{Subjects}

The two subjects were the author and a graduate student, both highly practiced in duration discrimination and ..duration reproduction experiments.

\begin{abstract}
Apparatus
The experiment was controlled on-line by a software control Wstem operating in a 4K Digital Equipment Corporation PDP-8/S computer. Subjects were run one at a time in a small isolated room partially lined with acoustic panels. The ambient noise level in the room was $51 \mathrm{~dB}$ SPL (A-weighting network), including masking noise provided by continuous operation of an air-conditioner fan. The subject listened to stimuli presented binaurally through a pair of Telephonics TDH-39 earphones. Each stimulus duration convisted of a silent interval bounded by a pair of clicks. The click "ituclorm was a 100-nicrosec square wave whose amplitude had heen previously adjusted to produce a comfortable listening level.

The stimulus durations were timed by a programmable clock controlled by the computer and driven by a $1,000-\mathrm{Hz}$ timebase. Onerall accuracy of timing was $\pm 1 \mathrm{msec}$.

The subject responded by pressing one of two push buttons. latheled " 1 " and " 2 ." which were located on a response box positioned on a table in front of him. A warning light was mounted on a panel behind the response box.
\end{abstract}

\section{Procedure}

A two-alternative forced-choice procedure was used in the experiment. Each trial began with a .1-sec flash of the warning light. Presentation of the standard $(\mathrm{T})$ and comparison $(\mathrm{T}+\Delta \mathrm{T})$ durations followed a $1-\mathrm{sec}$ foreperiod. The standard duration was alwars presented first. followed after a $2-\mathrm{sec}$ interstimulus interval by the comparison duration. The subject was instructed not to acount, tap, or in any other way to subdivide the presented intervals. The subject was required to choose the interval that he judged to be the longer of the two, indicating his choice by pressing the appropriate push button. No feedback was given concerning the correctness of his choice. Successive trials were separated by a 3-sec intertrial interval.

Fifteen values of the standard duration were chosen in the range from 5 () to $3,200 \mathrm{msec}$. Only one value of the standard was used in a given session. The order of use of the standards across the 15 sessions was randomly permuted for each subject.

The range of comparison durations to be used for each standard was determined in pilot sessions. A bounding duration, $\Delta T_{B}$. was determined for each standard such that the probability of a correct judgment was close to 1.0 when the standard $\mathrm{T}$ was compared with either $\mathrm{T}+\Delta T_{\mathrm{B}}$ or $\mathrm{T} \Delta T_{\mathrm{B}}$. A set of 11 comparison stimuli was then solected for each standard. spaced linearly through the range

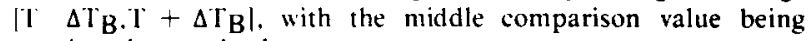
equal to the standard.

A session consisted of 30 block of 11 trials. each block consisting of a random permutation of the 11 comparison durations. The session length averaged about 45 min.

\section{RESULTS}

\section{Standard Deviation}

Psychometric functions were obtained for each standard duration for each subject by plotting the observed proportion of trials on which the comparison duration was judged to be longer as a function of the 
comparison duration. The observed proportions are function for the Weber's law model is approximately given for each subject in Table 1 .

cumulative normal. A normal ogive was fit to each

By assumption, the predicted psychometric function by transforming the set of proportions to $z$

Table 1

Proportion of Subject Judgments that Comparison is Longer

\begin{tabular}{|c|c|c|c|c|c|c|c|c|}
\hline \multirow{2}{*}{$\begin{array}{l}\text { Std } \\
\text { Comp } \\
\end{array}$} & \multicolumn{2}{|c|}{ Subject } & \multirow{2}{*}{$\begin{array}{l}\text { Std } \\
\text { Comp }\end{array}$} & \multicolumn{2}{|c|}{ Subject } & \multirow{2}{*}{$\begin{array}{l}\text { Std } \\
\text { Comp }\end{array}$} & \multicolumn{2}{|c|}{ Subject } \\
\hline & D.G. & T.W. & & D.G. & T.W. & & D.G. & T.W. \\
\hline 50 & & & 800 & & & 1800 & & \\
\hline 35 & .0 & .04 & 650 & .0 & .0 & 1600 & .0 & .0 \\
\hline 38 & .0 & .04 & 680 & .0 & .04 & 1640 & .04 & .12 \\
\hline 41 & .0 & .20 & 710 & .0 & .04 & 1680 & .0 & .12 \\
\hline 44 & .20 & .36 & 740 & .08 & .12 & 1720 & .20 & .40 \\
\hline 47 & .40 & .36 & 770 & .28 & .44 & 1760 & .16 & .44 \\
\hline 50 & .60 & .48 & 800 & .48 & .60 & 1800 & .72 & .48 \\
\hline 53 & .76 & .72 & 830 & .80 & .84 & 1840 & .84 & .60 \\
\hline 56 & 1.00 & .88 & 860 & 1.00 & 1.00 & 1880 & .76 & .88 \\
\hline 59 & .96 & .88 & 890 & 1.00 & 1.00 & 1920 & .92 & .88 \\
\hline 62 & 1.00 & .96 & 920 & 1.00 & .96 & 1960 & .96 & 1.00 \\
\hline 65 & 1.00 & 1.00 & 950 & 1.00 & 1.00 & 2000 & 1.00 & .96 \\
\hline 100 & & & 1000 & & & 2000 & & \\
\hline 75 & .0 & .0 & 900 & .0 & .0 & 1800 & .0 & .08 \\
\hline 80 & .04 & .12 & 920 & .0 & .08 & 1840 & .16 & .24 \\
\hline 85 & .0 & .04 & 940 & .12 & .24 & 1880 & .12 & .17 \\
\hline 90 & .04 & .24 & 960 & .28 & .24 & 1920 & .32 & .40 \\
\hline 95 & .36 & .48 & 980 & .68 & .52 & 1960 & .36 & .32 \\
\hline 100 & .52 & .64 & 1000 & .56 & .60 & 2000 & .52 & .64 \\
\hline 105 & .64 & .84 & 1020 & .88 & .84 & 2040 & .68 & .84 \\
\hline .110 & .96 & .88 & 1040 & .84 & .68 & 2080 & .80 & .76 \\
\hline 115 & 1.00 & 1.00 & 1060 & .88 & .88 & 2120 & .92 & .80 \\
\hline 120 & 1.00 & .96 & 1080 & 1.00 & .96 & 2160 & .96 & .88 \\
\hline 125 & 1.00 & 1.00 & 1100 & .96 & .96 & 2200 & 1.00 & 1.00 \\
\hline 200 & & & 1200 & & & 2400 & & \\
\hline 160 & .0 & .0 & 1075 & .0 & .08 & 1900 & .0 & .0 \\
\hline 168 & .0 & .0 & 1100 & .12 & .08 & 2000 & .04 & .0 \\
\hline 176 & .0 & .16 & 1125 & .16 & .36 & 2100 & .0 & .04 \\
\hline 184 & .12 & .16 & 1150 & .28 & .44 & 2200 & .16 & .64 \\
\hline 192 & .28 & .60 & 1175 & .28 & .72 & 2300 & .52 & .60 \\
\hline 200 & .72 & .64 & 1200 & .72 & .64 & 2400 & .84 & .92 \\
\hline 208 & .80 & .72 & 1225 & .56 & .88 & 2500 & .88 & .96 \\
\hline 216 & .96 & .84 & 1250 & .88 & .84 & 2600 & .96 & 1.00 \\
\hline 224 & 1.00 & .92 & 1275 & .88 & .96 & 2700 & .96 & 1.00 \\
\hline 232 & 1.00 & .96 & 1300 & .88 & 1.00 & 2800 & 1.00 & 1.00 \\
\hline 240 & 1.00 & .96 & 1325 & .96 & 1.00 & 2900 & 1.00 & 1.00 \\
\hline 400 & & & 1400 & & & 2800 & & \\
\hline 350 & .0 & .0 & 1250 & .04 & .0 & 2050 & .0 & .0 \\
\hline 360 & .0 & .0 & 1280 & .04 & .04 & 2200 & .0 & .16 \\
\hline 370 & .04 & .29 & 1310 & .16 & .20 & 2350 & .12 & .20 \\
\hline 380 & .04 & .44 & 1340 & .08 & .32 & 2500 & .12 & .20 \\
\hline 390 & .24 & .80 & 1370 & .36 & .64 & 2650 & .32 & .52 \\
\hline 400 & .68 & .84 & 1400 & .56 & .52 & 2800 & .54 & .68 \\
\hline 410 & .84 & .84 & 1430 & .60 & .84 & 2950 & .68 & .88 \\
\hline 420 & .92 & .92 & 1460 & .88 & .96 & 3100 & .96 & .96 \\
\hline 430 & .92 & 1.00 & 1490 & .80 & .92 & 3250 & 1.00 & 1.00 \\
\hline 440 & 1.00 & 1.00 & 1520 & 1.00 & 1.00 & 3400 & 1.00 & 1.00 \\
\hline 450 & 1.00 & 1.00 & 1550 & .88 & .96 & 3550 & 1.00 & 1.00 \\
\hline 600 & & & 1600 & & & 3200 & & \\
\hline 500 & .0 & .0 & 1400 & .0 & .04 & 2200 & .0 & .0 \\
\hline 520 & .0 & .0 & 1440 & .0 & .12 & 2400 & .04 & .0 \\
\hline 540 & .0 & .0 & 1480 & .12 & .16 & 2600 & .08 & .20 \\
\hline 560 & .08 & .08 & 1520 & .16 & .40 & 2800 & .24 & .32 \\
\hline 580 & .04 & .24 & 1560 & .44 & .48 & 3000 & .44 & .28 \\
\hline 600 & .64 & .52 & 1600 & .72 & .76 & 3200 & .44 & .50 \\
\hline 620 & .72 & .64 & 1640 & .76 & .96 & 3400 & .84 & .80 \\
\hline 640 & .96 & .80 & 1680 & .80 & .96 & 3600 & .92 & .88 \\
\hline 660 & .96 & .88 & 1720 & .96 & .92 & 3800 & .88 & .96 \\
\hline 680 & .96 & 1.00 & 1760 & .96 & 1.00 & 4000 & 1.00 & .96 \\
\hline 700 & 1.00 & 1.00 & 1800 & .96 & 1.00 & 4200 & 1.00 & .92 \\
\hline
\end{tabular}




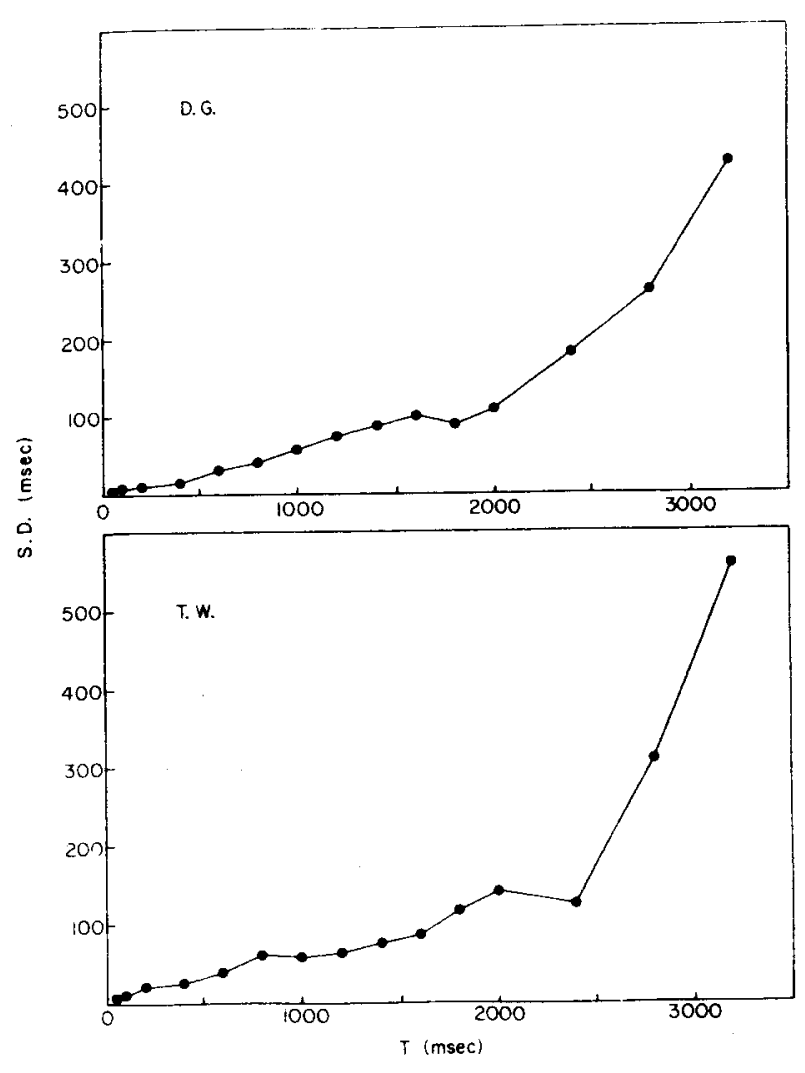

Figure 1. Estimated standard deviation of the psychometric density function for each subject as a function of the standard duration.

scores (excluding all proportions equal to zero or one) and then determining the best fitting straight line according to a least squares criterion. The normal ogive appears to provide an adequate approximation to all of the psychometric functions. The proportion of variance accounted for by the lines fit to the $z$ scores ranged from .836 to .999 , with an average of .924 across standards and subjects.

For each standard duration, the slope of the best titting line provides an estimate of the standard deviation of the psychometric density function while the $z$ intercept provides an estimate of the point of subjective equality (PSE). The estimates of standard deviation for each subject are shown in Figure 1 as a function of duration of the standard. The functions for both subjects exhibit a linearly increasing trend up to a standard duration of about $2 \mathrm{sec}$, after which the slope of both functions increases rapidly.

As noted earlier, the psychometric function predicted by the counter model is not precisely a symmetric normal ogive. Separate estimates of the standard deviation and PSE of each observed psychometric function were obtained for the counter model by varying its parameters to minimize the sum of squared deviations of the predicted from the observed points on the psychometric function. The standard deviations and PSEs estimated in this mamner are identical to, or within a few milliseconds of. those estimated by fitting normal ogives. For these data, the differences between the psychometric function predicted by the counter model and the normal ogive are extremely small.

\section{Weber Fraction}

The Weber fraction, $\mathrm{SD}(\mathrm{T}) / \mathrm{T}$, is plotted in Figure 2. For both subjects, the function initially drops over a short range of durations, is then flat over an extended range, and finally increases for long durations. This function is similar to that reported both by Blakely (1933) and by Stott (1933) except that the value of the Weber fraction observed here in the region of constancy is about .05 . This is considerably lower than their observed value of about .13, recomputed in terms of the standard deviation.

\section{Constant Error}

The constant error, PSE-T, is shown in Figure 3 as a function of the standard duration. The constant error for both subjects appears to be nearly 0 for small durations and to become increasingly negative, with positive acceleration, for increasing duration. A negative constant error signities that the comparison duration which the subject judges "equal" to a given standard duration is shorter than the standard. Since

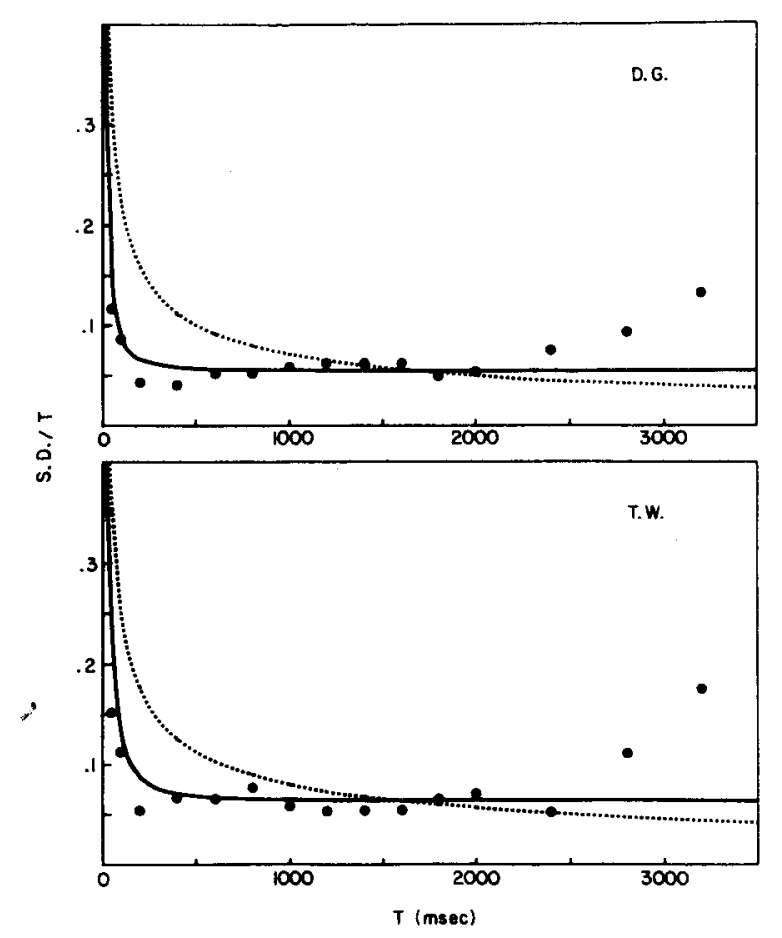

Figure 2. The Weber fraction, SD/T, obtained for each value of standard duration. The solid and dashed curves are the Weber fractions predicted by the Weber's law and counter models, respectively. 


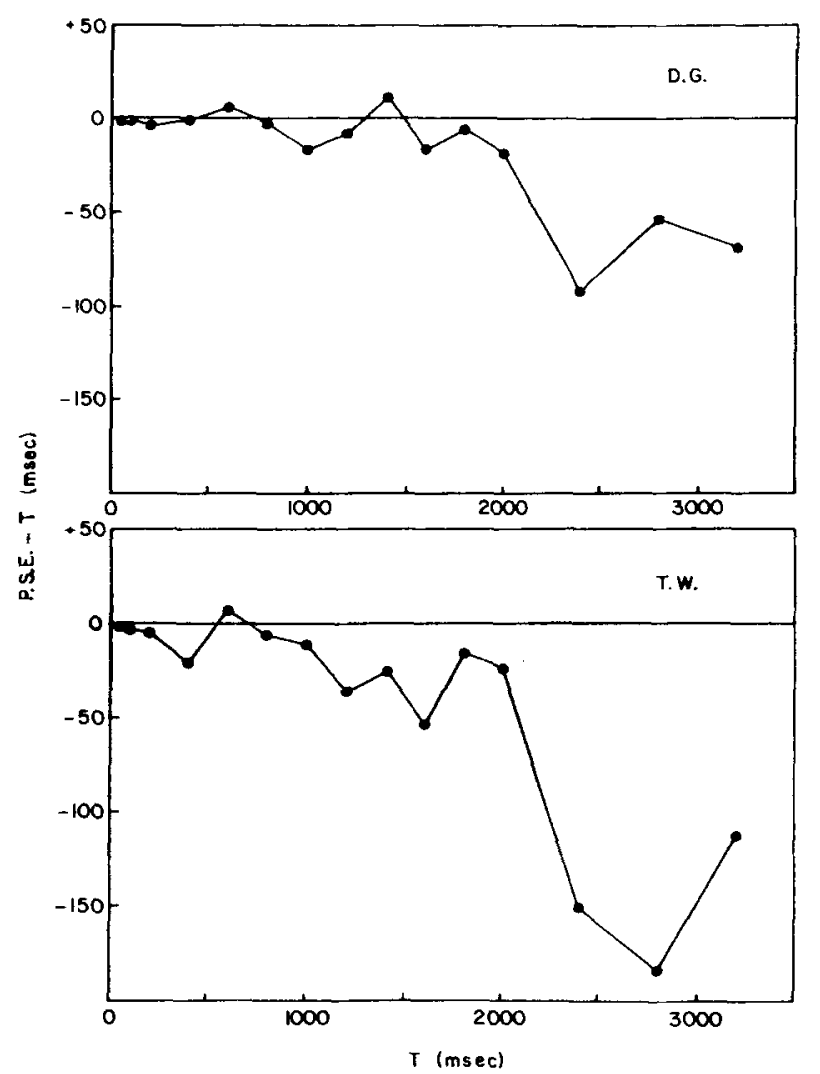

Figure 3. The constant error, PSE-T, as a function of the standard duration.

the comparison duration was always presented second in this experiment, a negative constant error corresponds to-negative time-order error (Woodrow, 1951). While neither model makes any specific prediction about the existence or type of time-order error, its occurrence in these results warrants further comment.

A number of duration-discrimination studies (Blakely, 1933; Stott, 1933, 1935; Woodrow, 1935) have reported negative time-order error for long durations. However, Allan and Kristofferson (1974) cite several recent studies (e.g., Allan, Kristofferson, \& Rice, 1974; Creelman, 1962) which found no duration-dependent time-order error. They suggest that the effect, when it occurs, may reflect response bias rather than a property of the perceptual process. The two results are not necessarily contradictory. The studies, including the present experiment, which have found duration-dependent time-order error have in common that no feedback was given the subject concerning the correctness of his response. Without feedback, the subject has no logical basis for altering his response bias to reduce time-order error. On the other hand, the studies reporting a lack of, or at least duration-independent, time-order error have generally utilized feedback. Over many trials, feedback provides sufficient information for a subject to shift his response criterion to minimize the increased error rate caused by time-order error. It is thus possible for a subject receiving feedback to compensate for time-order error, whatever its origin, resulting in little or no observable time-order error in his responses.

\section{MODEL EVALUATION}

Each model has both a slope parameter and a residual variance parameter to be estimated for each subject. The effect of the residual variance is most apparent for both models in the Weber fraction, where it produces an initial drop in the function. It is clear from the obtained Weber fraction (Figure 2) that the effect of the residual variance is negligible beyond a duration of about $400 \mathrm{msec}$. Consequently, this parameter was estimated using only the data for durations ranging from 50 to $400 \mathrm{msec}$. The use of data for longer durations would only contribute noise to the estimation procedure. Best fitting straight lines, using a least squares criterion, were determined according to Equation 3 for the Weber's law model and Equation 7 for the counter model.

Neither model can account for the rapid increase in standard deviation beyond a duration of $2 \mathrm{sec}$, since neither can predict the observed upward concavity for long durations. Consequently, the slope parameter was estimated for each model by fitting a straight line, with residual variance set to the value just estimated. to the data for durations from 50 to $2,000 \mathrm{msec}$. Again. Equations 3 and 7 were used for the Weber's law model and counter model, respectively.

The estimated parameters and the proportion of variance accounted for by each model are given for each subject in Table 2. In both cases, the Weber's law model accounts for a larger proportion of the variance in the data than the counter model. The fit of the Weber's law model is uniformly better than that of the counter model across durations. The absolute error in predicted standard deviation is smallest for the Weber's law model in 25 of the 30 comparisons for both subjects combined, a significant proportion $(p<.001$, sign test). The error in prediction made by

Table 2

Model Parameter Estimates for Each Subject and Proportion of Variance Accounted for by Each Model

\begin{tabular}{clrrr}
\hline Subject & \multicolumn{1}{c}{ Model } & $\mathrm{k}$ & $\mathrm{V}_{\mathbf{R}}$ & \multicolumn{1}{c}{$\begin{array}{c}\text { Propor- } \\
\text { tion of }\end{array}$} \\
\hline \multirow{2}{*}{ D.G. } & Weber's Law & .055 & 52 & .933 \\
& Counter & 4.942 & 4 & .875 \\
T.W. & Weber's Law & .064 & 141 & .922 \\
& Counter & 6.274 & $0^{*}$ & .741 \\
\hline
\end{tabular}

*The actual estimate was -10 . Since variance must be positive, the value was set to 0 . 


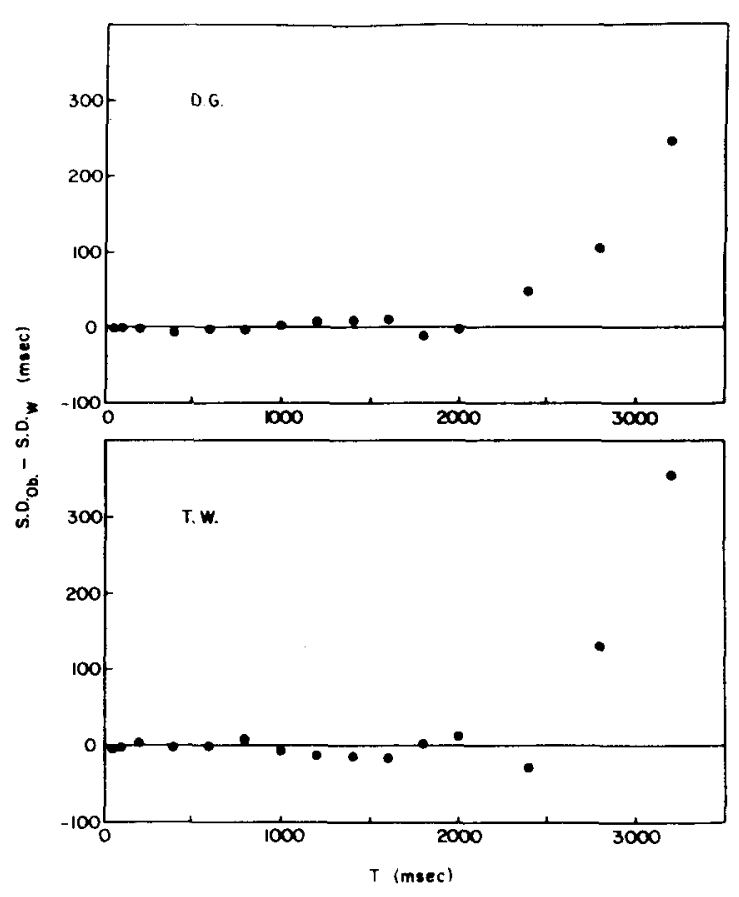

Figure 4. The error in predicted standard deviations, $S D_{O b}-S D_{w}$, for the Weber's law model as a function of standard duration.

the Weber's law model is shown in Figure 4 as a function of standard duration. Both the high quality of the lit for dirations less than about 2 sec and the fature of piediction for durations greater than $2 \mathrm{sec}$ ate erident.

Using the estimated parameter values, the Weber fraction was calculated for both the Weber's law model (Equation 5) and the counter model (Equition 9). The predicted curves are shown in Figure 2. The Weber's law model predicts the initial drop and subsequent constancy of the observed Weber fraction considerably better than the counter model. Neither model predicts the rise in the observed function for long durations.

\section{CONCLUSIONS}

The discrimination data from the present experiment are predicted significantly better by the Weber's law model than by the counter model. The results support Abel's (1972a) conclusion that the counter model does not provide an adequate description for durations longer than $100 \mathrm{msec}$. Moreover. the present results suggest that the Weber's law model may provide a better description of discrimination data than the counter model for durations below $100 \mathrm{msec}$ as well. However, this suggestion is made cautiously, since few very short durations were used in this experiment.
The results presented here are also not in accord with the predictions made by the onset-offset model of duration discrimination proposed by Allan et al. (1971). That model predicts that, within a range of duration values. the variance of the psychometric density function is a constant, independent of the standard duration. The smooth, essentially linear increase in standard deviation observed in the present experiment as a function of duration suggests that the underlying function is continuous and strictly monotone increasing. If there are steps of constant variance in the function, they must be quite small.

The Weber's law model fails to predict the more rapid increase in standard deviation for durations longer than $2 \mathrm{sec}$. The nature of the function for durations longer than $2 \mathrm{sec}$ is unclear from this study. However, for durations less than $2 \mathrm{sec}$, the Weber's law model accounts quite well for the observed relationship between standard deviation as an index of discriminability and duration.

It should be pointed out that while the counter model was specified in some detail, only one of its properties was used in its evaluation: namely, the predicted linear relationship between the variance of the psychometric density function and standard duration. Any other model which predicts a linear relationship between variance and duration, and whose predicted psychometric density function is normal. or nearly normal, would be equivalent to the counter model in terms of its ability to predict the observed standard deviations. Thus, the data provide evidence against the class of models which predict that the variance of the psychometric density function is a linear function of duration, and support for the class of models which predict that the standard deviation of the psychometric density function obeys Weber's law.

\section{REFERENCES}

ABEL, S. M. Duration discrimination of noise and tone bursts. Journal of the Acoustical Society of America, 1972, 51, 1219.1223. (a)

ABEL. S. M. Discrimination of temporal gaps. Joumal of the Acotustical Society of America. 1972, 52. 519-524. (b)

Allan, L. G., \& Kristofferson, A. B. Psychophysical theories of duration discrimination. Perception \& Psychophysics, 1974, 16. 26-34.

Allan. L. G., Kristofferson. A. B., \& Rice, M. E. Some aspects of perceptual coding of duration in visual duration discrimination. Perception \& Psychophysics, 1974, 15, 83-88.

Allan, L. G., Kristofferson, A. B., \& Wiens. E. W. Duration discrimination of brief light flashes. Perception \& Psychophysics, 1971. 9. 327-334.

Blakely. W. A. The discrimination of short empty temporal intervals. Unpublished doctoral dissertation. University of Illinois. 1933.

CreElman, C. D. Human discrimination of auditory durations. Journal of the Acoustical Society of America, 1962, 34. $582-59,3$. 
Holway, A. H., \& Pratt, C. C. The Weber-ratio for intensive discrimination. Psychological Review, 1936, 43, 322-340.

Kinchla, J. Duration discrimination of acoustically defined intervals in the 1- to 8-sec range. Perception \& Psychophysics, $1972,12,318-320$

Luce, R. D., \& Galanter, E. Discrimination. In R. D. Luce, R. R. Bush, and E. Galanter (Eds.), Handbook of mathematical psychology (Vol. I). New York: Wiley, 1963.

Mille R. G. A. Sensitivity to changes in the intensity of white noise and its relation to masking and loudness. Journal of the Acoustical Society of A merica, 1947, 19, 609-619.

Small, A. M. JR., \& Campbell, R. A. Temporal differential sensitivity for auditory stimuli. American Journal of Psychology, 1962, 75, 401.410.
Stotr, L. H. The discrimination of short tonal durations. Unpublished doctoral dissertation. University of Illinois. 1933.

Stotт, L. H. Time-order errors in the discrimination of short tonal durations. Journal of Experimental Psychology. $1935,18,741-760$

Woodrow. H. The effect of practice upon time-order errors in the comparison of temporal intervals. Psychological Review. 1935. 42. 127-152.

Woodrow, H. Time perception. In S. S. Stevens (Ed.), Handbook of experimental psychology. New York: Wiley, 1951.

(Received for publication December 6. 1974; revision received March 10, 1975.) 